\title{
Reviewer acknowledgement Journal of Polymer Engineering volume 39 (2019)
}

https://doi.org/10.1515/polyeng-2019-0310

The editors and the publisher wish to thank the following colleagues for their kind assistance in acting as referees for the journal this year: ${ }^{\star}$

Adekunle, K.

Ahmad, Ayaz

Ahmed, Shakeel

Akhter, Sohail

Al-Harthi, Mamdouh A.

Alhathal Alanezi, Adnan

Alikarami, Mohammad

Allahbakhsh, Ahmad

Almeida, Henrique A.

Alshdefat, Ramadan

Altstaedt, Volker

Alvarado-Iniesta, Alejandro

Amosa, Mutiu

Ang, Desmond Teck Chye

Arifin, Mohd. Azmir

Arora, Narinder

Askanian, Haroutioun

Atassi, Yomen

Azahari, B.

Azarian, Hossein

Barczewski, Mateusz

Basheer, A.

Beheshty, Mohammad Hosain

Benbettaieb, Nasreddine

Bijarimi, Mohd.

Bin Mohd Ali, Mohd. Amran

Brostow, Witold

Bucio, Emilio

Cacciotti, Ilaria

Cardinaels, Ruth

Carey, Jason

Carlone, Pierpaolo

Carneiro, Olga

Cha, Sung Woon

Chandrashekhara, K.

Cheng, Wei-Min

Chruściel, Jerzy J.

Colussi, Rosana

Cortez Tornello, Pablo R.

Cui, Xiaoping

D'Amato, Roberto

Danafar, Hossein

Darwish, M. S. A.

De Santis, Felice

Di Maio, Ernesto

Di Maio, Luciano

\author{
Di Profio, Gianluca \\ Dongre, Rajendra S. \\ Drioli, Enrico \\ Drstvensek, Igor \\ Elmaaty, T. A. \\ El-Sabbagh, Salwa \\ Fan, Tai-Hsi \\ Fernández Garcia, Marta \\ Filipe, Susana \\ Foot, Peter \\ Forero, Juan Carlos \\ Frolova, Liliya \\ Fuoco, Alessio \\ Gao, Aiqin \\ Gaspar-Cunha, António \\ Gedam, Asha H. \\ Giacomazza, Daniela \\ Gonçalves, Nelson \\ Gong, Jin \\ Grob, Koni \\ $\mathrm{Gu}$, Junwei \\ $\mathrm{Gu}$, Zhipeng \\ Gull, Nafisa \\ Gupta, Manoj \\ Gupta, Rajni \\ Gupta, Rakesh \\ Habi, Abderrahmane \\ Hade, Aaron \\ Hamouda, Tamer \\ Heli, Bentelhoda \\ Hrymak, Andrew \\ $\mathrm{Hu}$, Bin \\ $\mathrm{Hu}$, Zhanhu \\ Huang, Chao-Tsai \\ Huang, Congliang \\ Huang, Han-xiong \\ Huang, X. B. \\ Hyun, Kyu \\ Ianniruberto, Giovanni \\ Ilgin, Pinar \\ Itoh, Takahito \\ Jalali Dil, Ebrahim \\ Jia, Hongbing
}

\begin{abstract}
Jiang, Guohua
\end{abstract}
Johnson, Jed

Kalantari, Katayoon

Kallitsis, Joannis

Kaschta, Joachim

Kasuya, Kenichi

Kentaro, Iwashita

Kesavan, K.

Khajeheian, Mohammad Bagher

Khan, Aslam

Khan, Ijaz Ahmad

Khan, Mohammad

Khattak, Abraiz

Kleffel, Tobias

Ko, Y. G.

Koduru, H. K.

Kota, Sobha

Kühnert, Ines

Kumar, Rajiv

Kurniasih, Mardiyah

Kurose, Takashi

Lavorgna, Marno

Li, Shouhai

Li, Xiping

Lin, Haiqing

Liparoti, Sara

Lopez-Sanchez, Patricia

$\mathrm{Lu}, \mathrm{X}$.

Lucchetta, Giovanni

Luciano, Carla

Lucyshyn, Thomas

Luyt, Adriaan S.

Lv, Hongqing

M., Selva

Ma, Songqi

Mahdavi, Hossein

Manas, Miroslav

Manjunatha, C. M.

Masania, Kunal

Masek, Anna

Maximov, Anton

Mazidi, Majid Mehrabi

Mehmet, Bulut

Men, Yongfeng

Meng, Qingshi

Mensah, Alex Anim

* The list covers the time span October 5, 2018 to October 4, 2019. 
Mensitieri, Giuseppe

Mirza, M.

Miyata, Ken

Moayeri, Ali

Mollica, Francesco

Moon, Joshua

Motlagh, Ghodratollah Hashemi

Muenstedt, Helmut

Mukbaniani, Omari

Mulholland, Tom

Naderi, Ghasem

Nasseri, Behzad

Nicoletta, Fiore Pasquale

Nishitsuji, Shotaro

Nizami, A. S.

Nobile, Rossella

Nofar, Reza

Noori, Mohammad N.

Nunes, R. C. R.

Oh, Hee Jeung

Okimoto, Haruya

Onar Camlibel, Nurhan

Özgür, Cihan

P., Thirukumaran

Panin, Sergey V.

Park, Jin-Soo

Pasquino, Rossana

Pavlovic, Marko

Pilla, Srikanth

Pivsa-Art, Sommai

Ponikiewski, Tomasz

Portale, Giuseppe

Pourabbas, Behzad

Qi, Xiaowen

Qiao, Qiquan

R., Prithivirajan

Ragaert, Kim

Rahman, Ziyaur

Rana, Dipak
Read, Daniel

Redmann, Alec

Rezaei, Babak

Rupei, Tang

Sadak, Omer

Sadeghi, Morteza

Sahan, Nurten

Sahoo, Prasanta

Saleh, Tawfik

Salit, Mohd. Sapuan

Samra, Kawaljeet Singh

Sarasini, Fabrizio

Sarasua, Jose-Ramon

Sarti, Giulio

Schick, Christoph

Schroyen, Bram

Sedlařík, Vladimír

Shen, Jiangnan

Shi, Jianfeng

Shu, Dawu

Simon, Sara

Sinha, Ravi

Smet, Mario

Song, Yuyang

Soo Jin, Adrian Koh

Sorrentio, Andrea

Spalding, Mark

Speranza, Vito

Stifter, David

Strzemiecka, Beata

Subramaniam, Yugeswaran

Sugiman, Sugiman

Sultana, Saima

Sun, Weixing

Taboryski, Rafael

Takeuchi, Kenji

Takoudis, Christos G.

Talhi, Fatima Zohra

Tang, Rencheng

Tas, Mahmut

Tejero, Javier

Teke, Sengul
Teramoto, Hidetoshi

Then, Yoon Yee

Theravalappil, Rajesh

Todoroki, Akira

Tom, Engels

Tosello, Guido

Trzaskalska, Milena

Tseng, Shi-Chang

Turng, Lih-Sheng

Verma, Deepak

Vieru, Dumitru

Vipulanandan, C.

Vlachopoulos, John

Wang, Fangxin

Wang, Ping

Wang, Q.

Wang, Shi-Qing

With, Gijsbertus de

$\mathrm{Xu}, \mathrm{Fu}$

Yan, Ying

Yang, $Y$.

Yang, Yuqiu

Yao, Donggang

Yazdani-Ahmadabadi, H.

Yin, Teo

Yu, Houyong

Zapata, Paula A

Zatloukal, Martin

Zhang, Chaoqun

Zhang, Huihui

Zhang, L. Q.

Zhang, Lin

Zhang, Lu

Zhao, Changsheng

Zhao, Peng

Zhao, Ping

Zheng, Jinyang

Zhu, Shifeng

Zou, Hua 